\title{
ON TIME-FREE FUNCTIONS
}

\author{
BY \\ GIDEON SCHWARZ
}

\begin{abstract}
By regarding as equivalent any two real-valued functions of a real variable that can be obtained from each other by a monotone continuous transformation of the independent variable, time-free functions are defined. A convenient maximal invariant is presented, and applied to some time-free functional equations.
\end{abstract}

I. Introduction. In a earlier paper [9] we defined a time-free function as an equivalence class of continuous functions under composition with a time-scale transformation, that is, a homeomorphism of the independent variable. A matrix which is a maximal invariant for this equivalence relation was introduced there and was used to characterize a certain class of time-free (random) processes.

In $\S \mathrm{II}$ of this paper we carry out the constructions outlined in [9], and correct an error that was pointed out by Mr. Einhorn.

§III studies the solutions of the time-free functional equation $2 f \sim f$, and the functions fulfilling the more stringent requirement of binary time-free stability. Under a restriction to monotone functions, which is a point of view complementary to that of time-free functions, similar functional equations and conditions have been studied by Dubins and Savage [2] and by de Rham [7], [8].

In §IV we leave the time-free framework by introducing canonical time-scales, and study some consequences of binary time-free stability, including the metric dimensions of the graphs, a problem called to our attention by John Kinney. In defining metric dimension, we follow Kolmogorov [4] whose definition goes back to Pontrjagin and Schnirelman [6].

In $\S \mathrm{V}$ we outline some ideas on a class of time-free stochastic processes that seem to generalize Brownian motion in a natural way. So far we know very little about these processes.

I would like to thank Martin Fox and John Kinney for many inspiring discussions and Leo Katz who made it possible for me to complete this work at Michigan State University.

II. The matrix of a time-free function. Let $f$ be a real-valued unbounded continuous function on the nonnegative reals, having no interval in which it is constant, and taking on the value 0 at 0 . Associate with $f$ a matrix $A$, whose entries $a_{n j}$, defined for $n$ an integer and $j$ a positive integer, are all 1 or -1 , according to the following rule: $a_{n j}$ carries the sign of the $j$ th consecutive change of size $2^{-n}$ made by

Received by the editors September 12, 1970 .

AMS 1969 subject classifications. Primary 2630; Secondary 6062.

Copyright (c) 1972, American Mathematical Society 
the function. Thus the $n$th row of the matrix reflects the order in which $f$ performs up- and down-crossings of the intervals where $2^{n} f$ is between consecutive integers. Clearly $A$ cannot change when $f$ is composed with a continuous nondecreasing function mapping the nonnegative reals into themselves. So $A$ depends only on the time-free function of which $f$ is a representative.

Each row of the matrix $A$ determines the row above it: Group the entries of row $n$ into consecutive pairs. Any cancelling pair, that is, a pair of different entries, indicates a crossing of an interval of length $2^{-n}$, followed by a crossing of the same interval in the opposite direction. A pair of equal entries indicates consecutive crossings of two adjacent intervals of length $2^{-n}$ each, jointly comprising a single crossing, in the same direction, of an interval of length $2^{-(n-1)}$. Row $n-1$ of $A$ is therefore obtained by replacing the pairs of equal entries by the same entry taken once and ignoring cancelling pairs. We denote by $K$ the rule just described for obtaining from any row the row above it. $K$ is defined for any infinite sequence of \pm 1 's whose partial sums are unbounded, and it transforms it into another such sequence. For a matrix to be the matrix $A$ of some function $f$, it is necessary that all its rows $r_{n}$ fulfill this condition and that $K\left(r_{n}\right)=r_{n-1}$ for all $n$. In [9], these conditions were wrongly stated to be necessary and sufficient. Here is a counterexample: let $a_{n j}$, the $j$ th entry of the $n$th row of $A$, be -1 if $j<2^{n}$, and 1 otherwise. For $n \geqq 1$ the $n$th row has an initial sequence of $2^{n}-1$ minuses, followed by pluses only. These minuses form $2^{n-1}-1$ pairs, with one minus left over to form a single cancelling pair. Thus $K\left(r_{n}\right)=r_{n-1}$ holds. For $n \leqq 0, r_{n}$ contains only pluses and $K\left(r_{n}\right)=r_{n-1}$ trivially. Obviously the unboundedness condition is also fulfilled. However, if this $A$ were obtained from a function $f$, row $n$ with $n>0$, indicates that $f$ decreases at least as far down as $-2^{-n}\left(2^{n}-1\right)=-1+2^{-n}$ before it rises to, say, +1 . By continuity, it has to reach -1 before +1 . This contradicts the appearance of a plus at the beginning of row 0 .

This counterexample shows that $K\left(r_{n}\right)=r_{n-1}$ is not a sufficient condition for a matrix with "unbounded" rows of \pm 1 's to be obtained from a function $f$. Furthermore, since it is easy to find functions whose matrices will agree with the matrix of the counterexample on any finite set of rows, no condition that relates only finitely many rows to each other can be necessary and sufficient.

Consider the inverse image of a sequence $r$ under $K$. Its general element can be described as follows: when grouped into pairs of consecutive entries, the noncancelling pairs agree one by one with the elements of $r$. On cancelling pairs there is no restriction. Any noncancelling pair may be preceded by any finite string of $(-1,1)$ and $(1,-1)$ pairs in any order. We call that string, together with the noncancelling pair which it precedes the elaboration of the entry in $r$ to which that noncancelling pair corresponds. Thus the elaboration of $a_{n j}$ determines the sequence of steps of size $2^{-(n+1)}$ that make up the $j$ th step of size $2^{-n}$ of the function $f$. (In the counterexample above, the first plus of any row $r_{n}$ with $n \geqq 0$ has the elaboration $(-1,1,1,1)$. All other entries have the minimal elaboration, consisting of the 
entry repeated twice.) The sequence of entries of the $(n+k)$ th row, that determine the steps of size $2^{-(n+k)}$ that make up one particular step of size $2^{-n}$, is called the $k$ th elaboration of that step.

In the counterexample, there was a plus, namely $a_{01}$, whose $k$ th elaboration had for every $k$ some partial sum equal to $-\left(2^{k}-2\right)$. It was just this ingredient that made the counterexample work.

Proposition. The matrices $A$ obtained from functions are characterized by the following three properties:

(1) Each row is a sequence of \pm 1 's with unbounded partial sums.

(2) For every row $r_{n}, K\left(r_{n}\right)=r_{n-1}$.

(3) For every positive (negative) entry there is some $k$ such that the kth elaboration has no partial sum less than $-\left(2^{k}-2\right)$ (greater than $\left.2^{k}-2\right)$.

Furthermore, the set of functions corresponding to such a matrix constitutes exactly one time-free function.

Proof. Property (1) is equivalent to the unboundedness of the function.

The necessity of property (2) has been established before.

For the necessity of property (3), the argument explaining the counterexample applies after obvious adjustments.

The sufficiency will follow when for a given matrix $A$ with properties (1), (2) and (3), a function $f$ will be constructed such that $f$ gives rise to the matrix $A$. The following construction is outlined in [9]: Let $f^{0}$ be the function whose values at positive integers are the partial sums of row 0 of the matrix and whose values at noninteger points are filled in by linear interpolation, yielding a piecewise-linear, continuous function. Also, let $R_{0}$ denote the set of nonnegative integers. Having defined $f^{n}$ and $R_{n}$, consider for $j \geqq 1$ the elaboration of $a_{n j}$. It is a sequence of length, say, $k$, where $k$ is positive (and even). Divide the interval from the $(j-1)$ st to the $j$ th element of $R_{n}$ into $k$ equal parts. Adjoin the points of division obtained this way to $R_{n}$, obtaining a new set $R_{n+1}$. Now define $f^{n+1}$ as the function that takes on the partial sums of $2^{-(n+1)}\left(a_{n+1,1}, a_{n+1,2}, \ldots\right)$ at the points of $R_{n+1}$ in order, and is filled in by linear interpolation at all other points.

Property (2) ensures that $f^{n+1}$ agrees with $f^{n}$ on $R_{n}$. It is easily seen that nowhere can $f^{n+1}$ differ from $f^{n}$ by more than $3 \cdot 2^{-(n+1)}$, and therefore $f_{0}, f_{1}, \ldots$ converges to a continuous function $f$, which for every $n$ agrees with $f^{n}$ on $R_{n}$. Furthermore, the matrix $A_{n}$ which is obtained from $f^{n}$ agrees with $A$ in its $n$th row and hence on all previous rows. To violate this agreement in the limit, the $k$ th order elaborations of some positive (negative) entry of $A$ would have to have, for each $k$, at least one partial sum reaching the lowest (highest) possible value, and that is ruled out by condition (3). Therefore $f$ has $A$ as its matrix.

All that remains to be shown is that if $f$ and $g$ both give rise to the same matrix $A$, then $f$ and $g$ represent the same time-free function. It will suffice to show that for the case where $f$ is the function just constructed. Let $S_{n}$ be the set containing 
zero and the points at which $g$ completes consecutive steps of size $2^{-n}$, that is, the steps corresponding to the entries of $r_{n}$. Let $h_{n}$ be the function obtained by linear interpolation from the one-to-one order-preserving mapping of $S_{n}$ onto $R_{n}$. Each $h_{n}$ is strictly increasing from 0 to $\infty$, and continuous. On $S_{n}, f\left(h_{n}(\cdot)\right)$ agrees with $g$, and $h_{n+1}$ agrees with $h_{n}$. The absolute difference between $h_{n+1}$ and $h_{n}$ never exceeds the distance between adjacent points of $R_{n}$, which is at most $2^{-n}$. Therefore $h_{0}, h_{1}, \ldots$ approaches a continuous nondecreasing function $h$, and on $\bigcup S_{n}$ we have $f(h(\cdot))=g$. Since $g$ is nowhere constant, $\bigcup S_{n}$ is dense, $f(h(\cdot))=g$ holds everywhere and $h$ is strictly increasing. Q.E.D.

REMARKS. (1) It is possible to formulate condition (3) in terms of first-order elaborations only, by considering nested sequences of elaborations, in which each is an elaboration of an entry in the previous elaboration. (Since, however, there are uncountably many such sequences in a matrix, the measurability of condition (3) in the sigma-field generated by the entries would be hard to establish from that formulation, which comes up when random time-free functions are considered.)

(2) If the construction of $f$ is attempted for a matrix fulfilling conditions (1) and (2) but not (3), a continuous $f$ will be obtained, but its matrix will be different from the given one. This defines a natural mapping of such matrices into matrices fulfilling all three conditions.

III. Time-free binary stability. Clearly, if $A$ is the matrix of $f$, then the matrix of $2 f$ is obtained from $A$ by moving up all the rows of $A$, that is, replacing $r_{n}$ by $r_{n+1}$. Therefore $f$ and $2 f$ represent the same time-free function if and only if all the rows of $A$ are identical.

In order to find all such functions or, equivalently, in order to find the general solution of the functional equation $2 f(t)=f(h(t))$ where $h$ is an arbitrary function increasing strictly and continuously from 0 to $\infty$, the most general sequence $r$ of \pm 1 's for which $K(r)=r$ has to be found. The first two elements of $r$ can be chosen arbitrarily. Next, there comes an arbitrary, finite (but possibly empty) string of cancelling pairs followed by a noncancelling pair agreeing in sign with the first element of the sequence. This process continues, adding at the $m$ th stage an arbitrary string of cancelling pairs followed by a pair whose sign agrees with the sign of the $m$ th element of the sequence. The matrix $B$ all of whose rows equal the sequence thus constructed will have properties (1) and (2), but not necessarily (3). To ensure property (3), a complicated tail-property of the sequence is required, and we shall not go into details here. Anyway, in view of Remark (2), the general solution of $2 f(t)=f(h(t))$ can be described as the function constructed from $B$ as in the proof of the Proposition, composed with an arbitrary continuous function, strictly increasing from 0 to $\infty$.

Besides the functions $f(t)=t, f(t)=-t$ and their equivalence classes, the simplest example of a solution of the functional equation is given by the function that for $k=\ldots,-1,0,1, \ldots$ takes on the value $2^{k}$ at $2^{2 k}$ and 0 at $2^{2 k+1}$, with the rest filled 
in by linear interpolation. All rows of its matrix look like this: $1,-1,1,1,-1$, $-1,1,1,1,1,-1,-1,-1,-1, \ldots$ with the sequence obtained by starting out with $(1,-1)$ and choosing the empty string whenever the procedure calls for a string of cancelling pairs.

The class of solutions of the functional equation turned out to be quite rich. A smaller class, one that can be described much more explicitly, is obtained by replacing the functional equation by a stricter condition ${ }^{\prime}$ : rather than being content with $2 f$ being equivalent to $f$, we shall require that $f$ have the same time-free behaviour during any crossing of an interval of the form $\left(i 2^{-n},(i+1) 2^{-n}\right)$.

Definition. Let $f$ be a (continuous, unbounded, nowhere constant) function on the nonnegative reals, and let $I_{n j}=\left(\alpha_{n j}, \beta_{n j}\right)$ be the (time) interval on which $f$ makes its $j$ th step of size $2^{-n}$. Let $f_{n j}$ be the restriction of $f$ to $I_{n j}$. Then $f$ has the property of time-free binary stability if for every $n$ and $j$ we have $f_{n j}-f\left(\alpha_{n j}\right)= \pm f\left(h_{n j}(\cdot)\right)$, where $h_{n j}$ is a strictly increasing continuous mapping of $I_{01}$ onto $I_{n j}$, and the sign is chosen according to whether we have an up- or down-crossing.

Time-free binary stability is easily expressed in terms of the matrix $A$. It simply means that all positive entries have the same elaboration, and all negative entries have the elaboration obtained from that of the positive entries by replacing 1 by -1 and vice versa.

Now if the common elaboration of the positive entries of $A$ would contain a pair of the form $(-1,1)$, then some partial sum of this elaboration would equal -1 . The second-order elaboration would then have a partial sum equal to -3 and for every $k$ the $k$ th elaboration would have a partial sum equal to $-\left(2^{k}-1\right)$ and property (3) would be violated. Therefore the common elaboration of the positive entries must consist of, say, $m-1$ repetitions of the pair $(1,-1)$, followed by a single $(1,1)$-pair. Here $m$ can be any positive integer. For given $m$, only two matrices are possible. One whose general row begins with $m-1$ pairs $(1,-1)$, followed by a $(1,1)$-pair, and one with the opposite signs. Since now all partial sums of all elaborations of the positive (negative) entries are nonnegative (nonpositive), condition (3) holds and $A$ is the matrix of a function $f$. We denote by $f_{m}$ such a function when $m$ is given and the first entry is chosen positive. The set $\left\{ \pm f_{m} \mid m=1,2, \ldots\right\}$ now consists of one representative from each time-free binary-stable function. The matrix $A$ of $f_{m}$ can be expressed easily by using expansions of integers to the base $2 m$.

To find the entry $a_{n j}$, remove from the $(2 m)$-ary expansion of $j-1$ the digit $2 m-1$ whenever it occurs. If the sum of the remaining digits is odd, $a_{n j}=-1$; if it is even, $a_{n j}=1$.

Since $f_{m}$ makes $(2 m)^{n}$ steps of size $2^{-n}$ while making one unit step, it is of infinite variation in every interval when $m \geqq 2$. It is however of finite $\rho$-power variation, with $\rho=\log _{2}(2 m)$. No $f_{m}$ ever crosses zero (a global property, which does not seem to follow easily directly from time-free binary stability) though it does reach zero uncountably many times when $m \geqq 2$. 
IV. Non-time-free properties of the canonical $f_{m}$. The construction of a function from a matrix that occurs in the proof in §II yields a particular representative of the time-free function corresponding to the matrix. In the case of time-free binary stability this representative has a nice property that justifies calling it the canonical representative of $f_{m}$ : its $\rho$-power variation in every interval, with $\rho=\log _{2}(2 m)$, equals the length of the interval, that is, $\rho$-power variation is the canonical time scale for the $f_{m}$. That follows from the fact that in the construction each of the $(2 m)^{n}$ steps of size $2^{-n}$ that make up one unit step is allotted a time interval of length $(2 m)^{-n}$. We assume from here on that $f_{m}$ is the canonical representative and proceed to study some of its properties.

For the canonical case, the functional equation becomes $2 f_{m}(t)=f_{m}(2 m t)$. Therefore, the set $Z_{m}$ of zeros of $f_{m}$ is closed under multiplication and division by $2 m$. In $(2 m)$-ary notation, this means that $Z_{m}$ is closed under the shift operation. To find $Z_{m}$ we first find its intersection with the closed unit interval. The first approximation to $f_{m}$ in the canonical construction has 0 as its only zero in the unit interval. The zeros of the $n+$ first approximation in the unit interval are obtained from the zeros of the $n$th approximation by adding an arbitrary even digit on the left, and then shifting all the digits one position to the right. Observing that $Z_{m}$ is a closed set, one now easily obtains that all nonnegative reals that can be written $(2 m)$-arily with only even digits must be in $Z_{m}$. A similar argument, beginning with the observation that $f_{m}$ has no zeros in the open interval $(1,2)$, implies that $f_{m}$ has no other zeros: $Z_{m}$ is exactly the set of all nonnegative reals that have $(2 m)$-ary expansions containing only even digits.

Next, we find the function $\tau_{m}$, where, for $x \geqq 0, \tau_{m}(x)$ is the first $t$ such that $f_{m}(t)=x$. Let us borrow from decimal notation the digit 9 to denote $2 m-1$. We first find $\tau_{m}(x)$ for the positive integers. Since $\tau_{m}(1)=1$, the functional equation yields $\tau_{m}\left(2^{n}\right)=(2 m)^{n}$ for all $n$. Furthermore, since the last integer $t$ for which $f_{m}(t)=1$ before it goes higher is 9 , the last integer $t$ before $f_{m}(t)$ exceeds $2^{n}$ is $900 \ldots 00$ ( $n$ zeros). Since, in order to reach any integer $x, f_{m}$ must first reach the highest power of 2 which does not exceed $x$; then go up further by the highest power of 2 which does not exceed the remainder, etc.; we obtain the following prescription for $\tau_{m}(x)$ when $x$ is an integer: In the binary expansion of $x$, replace all 1's except the rightmost 1 by 9 . Then the result is the $(2 m)$-ary expansion of $\tau_{m}(x)$. Since $\tau_{m}$ fulfills the same functional equation as $f_{m}$, the prescription is valid for any binary rational $x$. To obtain $\tau_{m}(x)$ for all $x$, observe that by its definition as a hitting time $\tau_{m}(x)$ is left-continuous. Therefore the prescription is valid also when there is no rightmost 1 in the expansion of $x$. In fact, we can do away with the "rightmost 1 ", clause altogether: For all $x \geqq 0, \tau_{m}(x)$ is the number whose $(2 m)$-ary expansion is obtained from the nonterminating binary expansion of $x$ by replacing each 1 by $a$.

Next, we consider the graph of $f_{m}$ as a subset of the plane and find its metric dimension. Assume the part of the graph where $0 \leqq t \leqq 1$ is covered by $N_{\varepsilon}$ sets of diameter $<\varepsilon=(2 m)^{-n}$ each. Dividing the interval $0 \leqq t \leqq 1$ into $(2 m)^{n}$ equal parts, 
$(2 m)^{n}$ congruent parts of the graph are obtained. Each part has a diameter of at least $2^{-n}$ and must therefore intersect more than $2^{-n} /(2 m)^{-n}=m^{n}$ of the covering sets. If we count only the sets covering every other part, there can be no overlaps, and we obtain $N_{\varepsilon}>\frac{1}{2}(2 m)^{n} m^{n}$. On the other hand, the graph of $f_{m}$ over the unit interval can easily be covered by a unit square with sides parallel to the axes. Applying the functional equation $n$ times, the square is transformed into a rectangle that can be cut into $m^{n}$ squares of sidelength $(2 m)^{-n}$ and they would jointly cover the part of the graph over the interval $\left(0,(2 m)^{-n}\right)$. To cover the graph on the unit interval we therefore need $(2 m)^{n} m^{n}$ squares of diameter $\varepsilon^{\prime}=(2 m)^{-n} 2^{1 / 2}$. We obtain therefore

$$
\left(2 m^{2}\right)^{n} \geqq N_{\varepsilon^{\prime}} \quad \text { and } \quad N_{\varepsilon}>\frac{1}{2}(2 m)^{n} m^{n},
$$

which yields the metric dimension

$$
\alpha=\lim _{\varepsilon \rightarrow 0}\left(\log N_{\varepsilon} / \log \varepsilon^{-1}\right)=1+\log m / \log 2 m .
$$

In terms of the variation exponent $\rho=\log _{2}(2 m)$ defined earlier, we can write $\alpha=2-\rho^{-1}$. One should, however, keep in mind that $\rho$ is a time-free quantity, while $\alpha$ is valid for the canonical $f_{m}$ only. In fact, if instead of the canonical construction we use one in which the intervals are divided not into $2 m$ equal parts but according to some other fixed division, the resulting representative of $f_{m}$ has a graph whose metric dimension is strictly smaller than $\alpha$ : it is $2-H^{-1}$, where $H$ is the entropy of that fixed division which can be established by considerations in the spirit of McMillan's Asymptotic Equipartition Theorem (cf. e.g. [5]).

V. Some thoughts on the random case. When we go from time-free functions to random time-free functions, that is, to time-free processes, a natural generalization of the functional equation $2 f=f(h(\cdot))$ is the requirement that the time-free processes $X$ and $2 X$ have the same distribution. Passing from time-free functions to matrices, this becomes the requirement that all the rows of a random matrix have the same distribution, or equivalently, that the distribution of $r_{n}$ be invariant under the mapping $K$. The more stringent requirement of binary time-free stability can also be generalized for the random case by requiring all elaborations to be independent, with a common distribution for all elaborations of positive entries, and minus that distribution for the negative on $s$. The processes defined this way are in some ways similar to Dubins' and Freedman's random distribution functions [1], but the latter all reduce to the identity function from the time-free point of view.

The lengths $L_{k}$ of the $k$-order elaborations of some entry in row zero of the matrix of such a process form a Galton-Watson branching process with $\lambda=E\left(L_{1}\right)$ $\geqq 2$. If $E\left(L_{1}^{2}\right)$ is finite, $\lambda^{-n} L_{n}$ converges to a random variable, say $W$ (cf. [3, p. 13]). Defining $\rho=\log _{2} \lambda$, we have $\lambda^{-n}=\left(2^{-n}\right)^{\rho}$, and therefore $W$ is the (random) $\rho$-power variation of the path of the process during a unit step. One can therefore use $\rho$-power variation as a canonical time-scale. 
If the common distribution of all elaborations is chosen to be coin-tossing truncated after the first noncancelling pair, the process obtained has Brownian motion as its canonical representative. An equivalent formulation of that distribution is the following: choose the number of pairs in each elaboration according to a geometric distribution with parameter $p=\frac{1}{2}$, and then let each cancelling pair independently be $(-1,1)$ or $(1,-1)$ with probability $\frac{1}{2}$.

Choosing different values for $p$, we obtain a one-parameter class of time-free processes, which we call $B_{p}$. Here $\lambda=E\left(L_{1}\right)=2 / p$ and $\rho=1-\log _{2} p$, and the canonical versions of $B_{p}$ form generalizations of Brownian motion, "wiggling faster or slower" than Brownian motion according to whether $p<\frac{1}{2}$ or $p>\frac{1}{2}$. For $p=1$, we just obtain the identity function, and for the other extreme, $p=0, B_{p}$ is not defined.

\section{REFERENCES}

1. L. E. Dubins and D. A. Freedman, Random distribution functions, Bull. Amer. Math. Soc. 69 (1963), 548-551. MR 26 \#7008.

2. L. E. Dubins and L. J. Savage, How to gamble if you must. Inequalities for stochastic processes, McGraw-Hill, New York, 1965. MR 38 \#5276.

3. T. E. Harris, The theory of branching processes, Die Grundlehren der math. Wissenschaften, Band 119, Springer-Verlag, Berlin; Prentice-Hall, Englewood Cliffs, N. J., 1963. MR 29 \#664.

4. A. N. Kolmogorov and V. M. Tihomirov, $\varepsilon$-entropy and $\varepsilon$-capacity of sets in function spaces, Uspehi Mat. Nauk 14 (1959), no. 2 (86), 3-86. (Russian) MR 22 \#2890.

5. B. McMillan, The basic theorems of information theory, Ann. Math. Statist. 24 (1953), 196-219. MR 14, 1101.

6. L. S. Pontrjagin and L. G. Schnirelman, Sur un propriété métric de la dimension, Ann. of Math. 33 (1932), 156-162.

7. G. de Rham, Sur une courbe plane, J. Math. Pures Appl. (9) 35 (1956), 25-42. MR 19, 842.

8. - Sur quelques courbes definies par des équations fonctionnelles, Univ. e Politec. Torino, Rend. Sem. Mat. 16 (1956/57), 101-113. MR 20 \#1733.

9. G. Schwarz, Time-free continuous processes, Proc. Nat. Acad. Sci. U.S.A. 60 (1968), 1183-1188. MR 38 \#5280.

Department of Statistics, Michigan State University, East lansing, Michigan 48823

Institute of Mathematics, The Hebrew University of Jerusalem, Jerusalem, Israel 